\title{
Acute neurological problems: frequency, consultation patterns and the uses of a rapid access neurology clinic
}

\author{
${ }^{1}$ FA Chapman, ${ }^{2} \mathrm{AE}$ Pope, ${ }^{3} \mathrm{D}$ Sorensen, ${ }^{4} \mathrm{RSG}$ Knight, ${ }^{5} \mathrm{R}$ Al-Shahi Salman \\ ${ }^{1}$ Medical Student; ${ }^{2}$ Foundation Year Doctor, Medical School, University of Edinburgh; ${ }^{3}$ Information Analyst, NHS Lothian; ${ }^{4}$ Professor of \\ Clinical Neurology and Honorary Consultant Clinical Neurologist, Royal Infirmary of Edinburgh; ${ }^{5}$ MRC Clinician Scientist and Honorary \\ Consultant Neurologist, Division of Clinical Neurosciences, University of Edinburgh, Edinburgh, UK
}

ABSTRACT In secondary care, some patients with acute neurological symptoms are never seen by a neurologist. Rapid access neurology clinics could provide patients with timely access to neurology services. We analysed a retrospective cohort of 12,024 consecutive patients attending the 'immediate care' area of the emergency department or the acute medical admissions unit of the Royal Infirmary of Edinburgh. A total of I,036 patients $(9 \%)$ presented with a neurological complaint, of whom $680(66 \%)$ did not have any contact with neurology services. The most common problems were epileptic seizure, cerebrovascular diseases and headache. Of the patients with epileptic seizure or headache who were not seen by a neurologist, about $40 \%$ might have benefited from neurological assessment. Following the introduction of a weekly rapid access neurology clinic, the most common problems seen were headache, symptoms that turned out to be medically unexplained and epileptic seizure.

Published online December 2009

Correspondence to R Al-Shahi Salman, Bramwell Dott Building, Division of Clinical Neurosciences, Western General Hospital, Crewe Road South, Edinburgh EH4 2XU, UK

tel. +44 (0) I 3 I 5372944

e-mail Rustam.Al-Shahi@ed.ac.uk

KEYWORDS Accident and emergency, acute medicine, epilepsy, headache, neurology, stroke

DECLARATION OF INTERESTS No conflict of interests declared.

\section{INTRODUCTION}

Neurological problems are common in secondary care; they result in 3-8\% of emergency department attendances, 15-20\% of medical unit admissions, $40 \%$ of inpatients on medical wards and many requests for inpatient consultation. ${ }^{1-7}$

Studies have demonstrated the value of neurologists in confirming and making de novo neurological diagnoses, in revoking or changing at least one fifth of emergency or general physicians' neurological diagnoses and in reducing patients' length of stay in hospital. ${ }^{4,8-14}$

The long-term aspiration of the Association of British Neurologists is for all patients presenting to hospital with acute neurological problems to be assessed immediately by a neurologist. ${ }^{8}$ However, most of these patients are never seen by a neurologist, and tend to be managed by general physicians.,

A rapid access neurology clinic is one interim solution that could help manage patients with semi-urgent neurological problems who do not require admission to hospital. The rationale is that this clinic would enable patients who contact emergency services with neurological problems to be managed quickly by neurologists, potentially reducing hospital admissions ${ }^{15}$ and targeting investigations appropriately. This service model seemed to be ideally suited to the workforce and geographical limitations in Edinburgh, where there is no

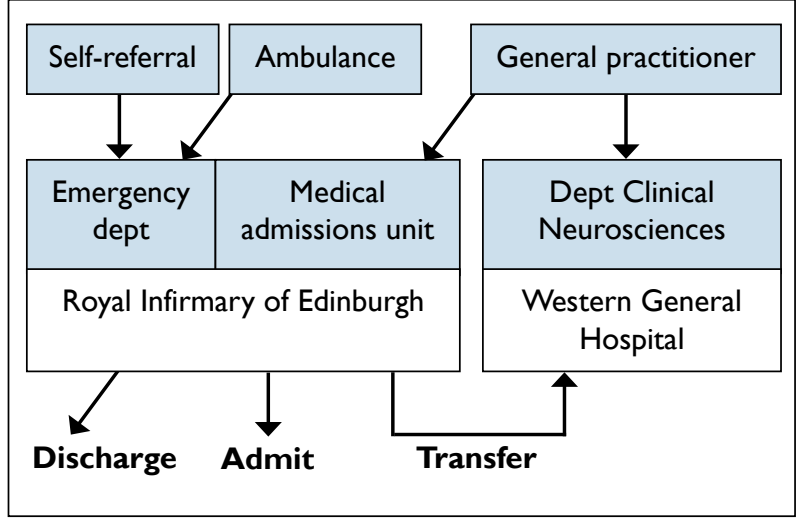

FIGURE I Access to emergency medical services for citizens of Edinburgh with acute neurological problems in the last quarter of 2005.

on-site neurology service at the hospital housing the regional emergency department.

\section{METHODS}

\section{Study setting}

NHS Lothian delivers healthcare to a population of about 800,000 . The $\sim 380,000$ adults living in Edinburgh who are the focus of this study are served by the Emergency Department at the Royal Infirmary of Edinburgh (RIE). At the time of this study in 2005, patients with acute neurological problems could obtain urgent assessment via several routes (Figure I). Protocols were available to guide the management of some neurological conditions 
for which dedicated services existed, such as transient ischaemic attack or stroke, and first seizure. However, some patients with neurological problems seen in the Emergency Department or the RIE's Combined Assessment Unit (medical admissions) may not have been admitted by, discussed with or followed up by neurologists at the regional neuroscience centre based at the Western General Hospital, six miles away.

\section{Cross-sectional service evaluation of patients attending} the emergency department or medical admissions unit

We retrospectively searched electronic health records systems at the RIE to identify all attendances in the Immediate Care ('majors') area of the Emergency Department and the GP-referral area ('trolleys') of the medical admissions unit from I October 2005 until 3 | December 2005 inclusive. AE Pope and FA Chapman (and R Al-Shahi Salman in cases of doubt) reviewed the entire free-text record of each Emergency Department attendance or the diagnostic coding of each medical admissions unit attendance (with reference to the entire electronic patient record when necessary) or both. ${ }^{16}$ We used the patient record to: categorise the principal reason for each attendance as neurological, neurosurgical or other; sub-classify the neurological diagnoses; determine whether a member of the neurology service was contacted; and ascertain whether patients were admitted or followed up by neurology services. ${ }^{17}$

Patients with neurological problems but no contact with the neurology service

In order to ascertain the greatest potential demand for a rapid access neurology clinic, we identified patients with neurological problems who were not documented as being referred to on-call, inpatient or outpatient neurology services. R Al-Shahi Salman further scrutinised the two most common diagnoses in this group (epilepsy and headache) to determine which patients might have been likely to benefit from a rapid access neurology clinic (for example, poorly controlled epilepsy, poorly controlled migraine, cases of diagnostic doubt, cases where investigation was undertaken but seemed unnecessary or headache/epilepsy resulting in hospital admission) or not (for example, alcohol withdrawal seizures and resolved headache).

\section{Evaluation of the Rapid Access Neurology Clinic}

This service evaluation led to the creation of a Rapid Access Neurology Clinic (RANC) on 5 January 2007, run by two consultant neurologists (R Al-Shahi Salman and RSG Knight) every Friday morning at the Royal Infirmary of Edinburgh. The RANC has six half-hour slots for patients seen with neurological problems in the Emergency Department or medical admissions unit over the preceding week, who are not already under the care of a consultant neurologist and whose problem is not appropriate for existing dedicated, protocol-directed services (first seizure, neurovascular and syncope).

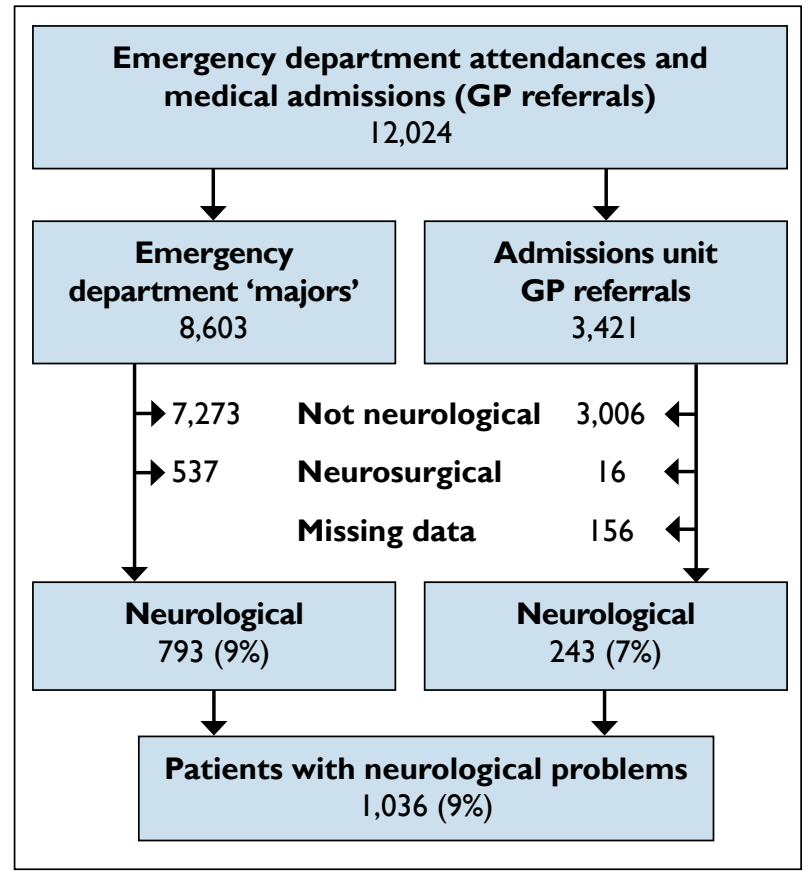

FIGURE 2 Neurological problems among all attendances in unscheduled care over three months.

Patients are booked into the clinic at the discretion of the doctor assessing them (sometimes after discussion with the on-call neurology service to determine if the patient would benefit from semi-urgent neurological assessment). The RANC is also accessible to the Emergency Department at St John's Hospital in Livingston so that service provision is equitable in Lothian (the administrative region in which Edinburgh is located). FA Chapman retrospectively assessed the characteristics of the patients seen in the first year of the RANC, using the electronic health records system, and applied the same diagnostic sub-classifications as had been applied to the retrospective cohort.

This project involved service evaluation, audit and quality improvement, so research ethics committee approval was not required.

\section{RESULTS}

Patients attending the emergency department or medical admissions unit

Of 12,024 patient attendances over three months, $9 \%$ overall were primarily due to a neurological problem (Figure 2). The most common diagnoses were epileptic seizure(s), cerebrovascular diseases and headache (Table I). Of these I,036 patients, neurology or neurosurgery services were consulted about 88 cases $(8 \%)$ and a further 268 (26\%) reached an appropriate service involving neurologists. Where either consultation with, or follow-up by, neurological services was not specified, we assumed they had not taken place, leaving 680 patients $(66 \%)$ without any apparent contact with neurological services (Table 2 overleaf). 
TABLE I Diagnostic breakdown of I,036 patients attending unscheduled care services with neurological problems, subdivided into those who were discussed with neurological services and those who were not

\begin{tabular}{|c|c|c|c|}
\hline \multirow[t]{2}{*}{ Diagnostic category } & \multicolumn{3}{|c|}{ Number (\%) of patients } \\
\hline & Total & Consulted & $\begin{array}{l}\text { Not } \\
\text { consulted }\end{array}$ \\
\hline Epileptic seizure(s) & $363(35 \%)$ & 128 & 235 \\
\hline $\begin{array}{l}\text { Transient ischaemic } \\
\text { attack and stroke }\end{array}$ & $218(21 \%)$ & 150 & 68 \\
\hline Headache & $14 \mid(14 \%)$ & 21 & 120 \\
\hline $\begin{array}{l}\text { Delirium and neuro- } \\
\text { degenerative disorders }\end{array}$ & 69 (7\%) & 7 & 62 \\
\hline Vestibular disorders & $67(6 \%)$ & 5 & 62 \\
\hline $\begin{array}{l}\text { Non-specific } \\
\text { neurological symptoms }\end{array}$ & $60(6 \%)$ & II & 49 \\
\hline Spinal disorders & $31(3 \%)$ & 2 & 29 \\
\hline $\begin{array}{l}\text { Neuromuscular } \\
\text { disorders and cranial } \\
\text { neuropathies }\end{array}$ & $25(2 \%)$ & 7 & 18 \\
\hline $\begin{array}{l}\text { Medically unexplained } \\
\text { symptoms }\end{array}$ & $17(2 \%)$ & 8 & 9 \\
\hline $\begin{array}{l}\text { Subarachnoid } \\
\text { haemorrhage }\end{array}$ & $8(1 \%)$ & 8 & 0 \\
\hline $\begin{array}{l}\text { Neurological } \\
\text { infections }\end{array}$ & $7(1 \%)$ & 2 & 5 \\
\hline Tumours & $7(1 \%)$ & 2 & 5 \\
\hline Functional disorders & $6(<1 \%)$ & I & 5 \\
\hline Movement disorders & $5(<1 \%)$ & 1 & 4 \\
\hline Pain syndromes & $4(<1 \%)$ & I & 3 \\
\hline Coma & $3(<1 \%)$ & I & 2 \\
\hline Multiple sclerosis & $3(<1 \%)$ & I & 2 \\
\hline Trauma & $2(<1 \%)$ & 0 & 2 \\
\hline Total & 1,036 & 356 & 680 \\
\hline
\end{tabular}

Patients with neurological problems but no contact with the neurology service

A mean of 52 patients per week over three months did not have any contact with neurological services, and they accounted for a sizeable burden on general medical services: $286(42 \%)$ were admitted to the medical admissions unit, and 34 (5\%) were followed up in medical outpatients. The majority of the patients without contact with neurology services had presented with epileptic seizure(s) or headache (Table I). One author (R Al-Shahi Salman) reviewed these 355 patients' medical records, and found I 42 (40\%, mean II patients per week) might have benefited from neurological consultation (as described in the Methods section): 105 (30\%) were admitted, of whom 62 (59\%) might have benefited, and $250(70 \%)$ were discharged, of whom $80(32 \%)$ might have benefited.
TABLE 2 Outcome of patient attendance, cross-tabulated against whether there had been consultation with neurology or neurosurgery

\begin{tabular}{|c|c|c|c|c|}
\hline & $\begin{array}{l}\text { Neurology } \\
\text { consults } \\
(83)\end{array}$ & $\begin{array}{l}\text { Neuro- } \\
\text { surgery } \\
\text { consult } \\
(5)\end{array}$ & $\begin{array}{l}\text { No } \\
\text { neurology } \\
\text { consult } \\
(890)\end{array}$ & $\begin{array}{l}\text { Unknown } \\
\text { consult } \\
(58)\end{array}$ \\
\hline \multicolumn{5}{|c|}{ Using appropriate or existing neurology services } \\
\hline $\begin{array}{l}\text { Admit to } \\
\text { neurology }\end{array}$ & $17(20 \%)$ & - & - & - \\
\hline $\begin{array}{l}\text { Admit to } \\
\text { stroke unit }\end{array}$ & $2(2 \%)$ & - & $92^{\S}(10 \%)$ & - \\
\hline $\begin{array}{l}\text { Urgent } \\
\text { neurology } \\
\text { outpatient }\end{array}$ & $7(9 \%)$ & - & $2^{\S}(1 \%)$ & - \\
\hline $\begin{array}{l}\text { Routine } \\
\text { neurology } \\
\text { outpatient }\end{array}$ & $13(16 \%)$ & $\mathrm{I}(20 \%)$ & $47^{\S}(5 \%)$ & - \\
\hline $\begin{array}{l}\text { First seizure } \\
\text { clinic }\end{array}$ & $5(6 \%)$ & - & $56^{\S}(6 \%)$ & $\mathrm{I}(2 \%)$ \\
\hline $\begin{array}{l}\text { Neurovascular } \\
\text { outpatient }\end{array}$ & $2(2 \%)$ & - & $54 \S(6 \%)$ & - \\
\hline $\begin{array}{l}\text { Admit to } \\
\text { critical care }\end{array}$ & $2(2 \%)$ & $\mathrm{I}(20 \%)$ & $12(1 \%)$ & $4(7 \%)$ \\
\hline \multicolumn{5}{|c|}{ Using non-neurological services } \\
\hline $\begin{array}{l}\text { Admit medical } \\
\text { admissions unit }\end{array}$ & $19(23 \%)$ & $2(40 \%)$ & $\begin{array}{l}254^{*} \\
(29 \%)\end{array}$ & $32 *(55 \%)$ \\
\hline $\begin{array}{l}\text { Medical } \\
\text { outpatients }\end{array}$ & $\mathrm{I}(\mathrm{I} \%)$ & I (20\%) & $34^{*}(4 \%)$ & - \\
\hline No follow-up & $7(9 \%)$ & - & $\begin{array}{l}328 \\
(37 \%)\end{array}$ & $2 *(3 \%)$ \\
\hline Unknown & $8(10 \%)$ & - & $1 \mathrm{I}^{*}(1 \%)$ & $19^{*}(33 \%)$ \\
\hline
\end{tabular}

${ }^{\S}$ Direct referral to these existing services was possible, without the need for consultation

${ }^{*}$ Shaded boxes $=$ no apparent contact with neurology services $(n=680$, $66 \%$ of total of 1,036 patients)

\section{Evaluation of the Rapid Access Neurology Clinic}

During its first year, 167 patients were referred to the RANC; I54 patients attended their appointment, within a median of two days of their initial presentation. Referrals to the clinic were slow to accumulate, but by the end of the year all six new patient slots were being used. In comparison to the neurological problems that did not result in neurological consultation in the threemonth service evaluation, in the first year of the RANC headache and medically unexplained symptoms constituted a greater proportion and epileptic seizure(s) a smaller proportion of the workload (Figure 3). We did not formally assess whether admissions and length of stay were reduced in a before-and-after study; conversely, three patients who were referred to the RANC were admitted following their appointments. 


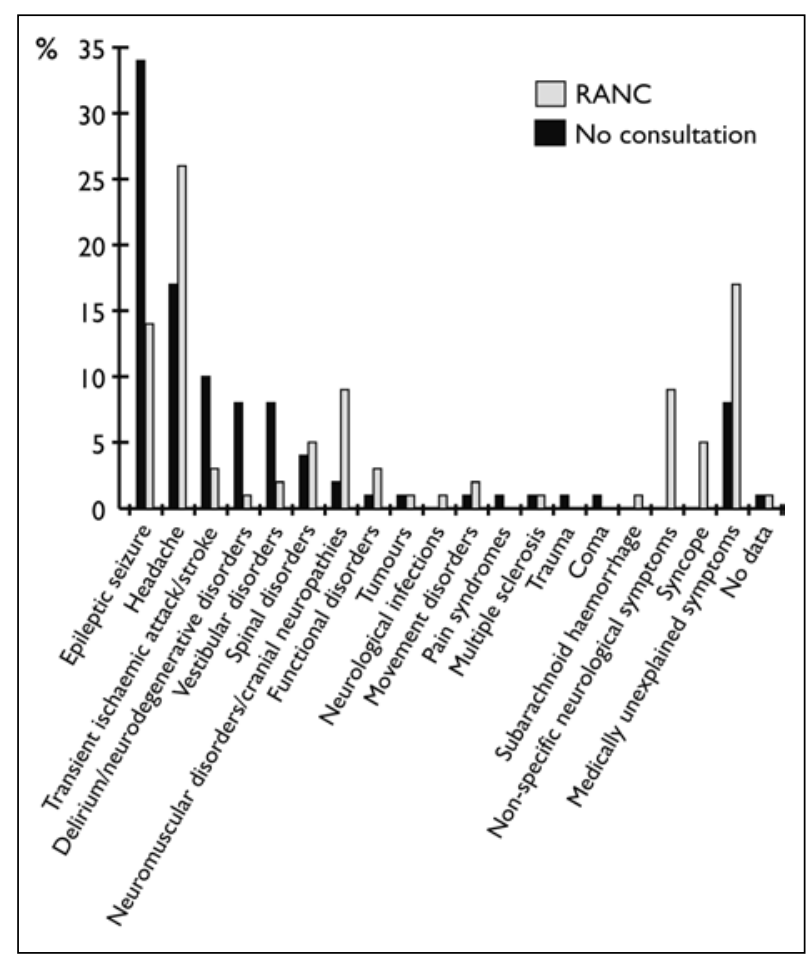

FIGURE 3 Frequency of neurological diagnoses among patients who did not have contact with neurological services in the three-month service evaluation (black) and among patients who were referred to the Rapid Access Neurology Clinic in its first year (grey).

\section{DISCUSSION}

In a three-month study of I2,024 consecutive emergency attendances at the RIE, $9 \%$ were due to neurological problems. ${ }^{1,2}$ Epileptic seizure(s), cerebrovascular diseases and headache were the three leading neurological problems. We found that two thirds of patients presenting with neurological problems were not discussed with, admitted or followed up by a neurologist; these numbered a mean of 52 such patients per week, of whom $42 \%$ were admitted to the medical admissions unit. Of the patients with epileptic seizure(s) and headache who were not seen by a neurologist, $40 \%$ might have benefited from a neurological assessment.

There may have been several reasons why neurologists were not involved with the majority of patients with neurological problems. We found that neurological assessment would have been unlikely to be helpful for $60 \%$ of the patients with headache or epileptic seizure(s), and this may have applied to a greater or lesser extent to the other diagnoses. General and emergency physicians are capable of managing some neurological emergencies, and had recourse to protocols for first seizures, transient ischaemic attack and stroke at the time of this study. However, we found room for improvement in the management of the patients with sudden severe headache in this series, leading us to create a protocol for their management. ${ }^{18}$ Alternative explanations for the paucity of referrals include the pressure on emergency physicians to meet waiting-time targets (which might encourage discharge without discussion) and the location of the neuroscience centre in a different hospital.

This study has strengths and weaknesses. We included $>12,000$ patients, which meant that most acute neurological problems were represented (Table I). Only I56 (I\%) patients' data were missing. Although our findings are generalisable to other settings where the neuroscience centre is located in a different hospital from the emergency department, we will have underestimated the incidence of acute neurological problems because many patients with these problems will have been referred direct to the neuroscience centre. Because this study investigated the neurological problems not referred to neurologists, we had to rely on emergency and general physicians' clinical descriptions and diagnostic categorisations. Ideally, the impact of the RANC should be assessed in a before-and-after study, by repeating our initial three-month audit of all acute neurological problems in the era of the RANC.

This study has implications for those managing and working in acute medicine. From just a review of patients with headache and epileptic seizure(s) who had not been referred to neurology services, $30 \%$ of these patients were admitted to hospital, of whom $59 \%$ might have benefited from a neurological opinion. It is plausible that a neurologist's involvement might have avoided some of these admissions, especially in the light of a systematic review, which found that up to one fifth of UK hospital admissions are inappropriate. ${ }^{19}$ From a local perspective, co-locating the neuroscience centre with the emergency department at the RIE should further involve neurologists in the care of patients with neurological problems in unscheduled care.

Until the consultant neurologist workforce in the UK has expanded sufficiently to assess every patient with an acute neurological problem within 24-48 hours, initiatives such as rapid access neurology clinics could help neurologists 'engage in the day-to-day business of district general hospitals', improve patient care and even minimise hospital admissions and re-attendances. ${ }^{20}$

Acknowledgements We are indebted to Aidan Hutchison for his programming expertise. We are grateful to Annette Henderson and Ross Davies of NHS Lothian's Clinical Effectiveness department for helping with data collection for this service evaluation. 


\section{REFERENCES}

I García-Ramos R, Moreno T, Camacho A et al. [Neurological emergencies in a university hospital.] Neurologia 2003; 18:43I-8. In Spanish.

2 Craig J, Patterson V, Roche L et al. Accident and emergency neurology: time for a reappraisal? Health Trends 1997; 29:89-9I.

3 Morrow Jl, Patterson VH. The neurological practice of a district general hospital. J Neurol Neurosurg Psychiatry 1987; 50:1397-40I. doi:I0.I I36/jnnp.50.II.1397

4 Forbes R, Craig J, Callender $M$ et al. Liaison neurology for acute medical admissions. Clin Med 2004; 4:290.

5 Weatherall MW. Acute neurology in a twenty-first century district general hospital.J R Coll Physicians Edinb 2006; 36:196-200.

6 Playford ED, Crawford P, Munro PS. A survey of neurologica disability at a district general hospital. Br J Clin Pract 1994; 48:304-6.

7 Barrero-Hernández FJ, Muñuzuri-Sanz D, Casado-Torres A. [A descriptive study of intrahospital neurology service consultations.] Rev Neurol 2003; 36: I00I-4. In Spanish.

8 Association of British Neurologists. Acute neurological emergencies in adults. London: Association of British Neurologists; 2002.

9 Steiger MJ, Enevoldson TP, Hammans SR et al. Influence of obtaining a neurological opinion on the diagnosis and management of hospital in-patients. J Neurol Neurosurg Psychiatry 1996; 61:653-4. doi:I0.1 136/ jnnp.6I.6.653-a

10 Costello DJ, Renganathan R, O'Hare A et al. Audit of an inpatient neurology consultation service in a tertiary referral centre: value of the consulting neurologist. Ir Med J 2005; 98:134-7.

I I Moeller JJ, Kurniawan J, Gubitz GJ et al. Diagnostic accuracy of neurological problems in the emergency department. Can J Neurol Sci 2008; 35:335-4I.
I2 Hillen ME, Sage JL. Proving the worth of neurologists. Neurology 1996; 46:276-7.

13 Nixon J, Bateman DE, Malthouse S. Computerised tomography scans of the head in a district general hospital. J $R$ Coll Physicians Lond 1996; 30:547-50.

I4 Moulin T, Berger E, Lemounaud P et al. [Emergency consultations in the university hospital setting: contribution of the neurologist to inpatient management.] Rev Neurol (Paris) 2000; 156:727-35. In French.

I5 Purdy S, Griffin T. Reducing hospital admissions. BMJ 2008; 336:4-5. doi:I0.I I36/bmj.39394.402465.BE

16 World Health Organization. ICD-I0: the ICD-I0 classification of mental and behavioural disorders; diagnostic criteria for research. 10th revision. Geneva:World Health Organization; 1993.

17 Association of British Neurologists. Neurology in the United Kingdom: towards 2000 and beyond. London: Association of British Neurologists; 1997.

I8 Breen DP, Duncan CW, Pope AE et al. Emergency department evaluation of sudden, severe headache. QJM 2008; 101:435-43. doi: I0.1093/qjmed/hcn036

19 McDonagh M,S Smith DH, Goddard M. Measuring appropriate use of acute beds. A systematic review of methods and results. Health Policy 2000; 53:I57-84. doi:I0.I0I6/S0I68-85I0(00)00092-0

20 Warlow C, Humphrey P, Venables G. UK neurologists and the care of adults with acute neurological problems. Clin Med 2002; 2:436-9.

\section{EDITORIAL VACANCY AT THE JOURNAL}

\section{History Editor}

\section{The Journal of the Royal College of Physicians of Edinburgh}

Expressions of interest are invited for the above post from Fellows or Members of the College. The position is honorary and will become available in January 2010, for a term of two years. Support is provided by an experienced production staff of three within the College.

The JRCPE is a quarterly, 96-page publication consisting of three sections - Clinical, Education and History. It has a circulation of about 8,000, including all Fellows and Collegiate Members and some postgraduate libraries. The History Editor will be working together with the Editor, Assistant Editor and Clinical Editor.

The History Editor is responsible for selecting appropriate referees, revising/editing and selecting/rejecting submitted papers for the History section of the JRCPE (approx. 25 pages per quarter). He or she will aim to achieve a high standard of published articles and encourage submission wherever possible to ensure an adequate flow of papers.

Applicants for the post will require good communication skills, a knowledge of the writing and editing of scientific articles, an interest in medical history and sufficient time to deal promptly with editorial decisions.
For further information, please contact:

Graeme McAlister, Head of Fellowship and Membership Services, Royal College of Physicians of Edinburgh, 9 Queen Street, Edinburgh EH2 IJQ, UK

Tel: +44 (0) I 3 I 2473693 Email: g.mcalister@rcpe.ac.uk A full job description is available online at: www.rcpe.ac.uk (under 'What's new') Closing date: 15 January 2010 